\title{
Autobiographies de la recomposition : esthétique d'une écriture conflictuelle dans l'œuvre de Barbara Balzerani
}

Autobiografie della ricomposizione: estetica di una scrittura conflittuale

nell'opera di Barbara Balzerani

Autobiographies of Recomposition: Aesthetics of Conflictual Writing in Barbara

Balzerani's Literary Work

Marie Thirion

\section{OpenEdition}

Journals

Édition électronique

URL : https://journals.openedition.org/cei/8832

DOI : 10.4000/cei.8832

ISSN : 2260-779X

Éditeur

UGA Éditions/Université Grenoble Alpes

Édition imprimée

ISBN : 978-2-37747-257-4

ISSN : 1770-9571

Référence électronique

Marie Thirion, «Autobiographies de la recomposition : esthétique d'une écriture conflictuelle dans l'œuvre de Barbara Balzerani », Cahiers d'études italiennes [En ligne], 32 | 2021, mis en ligne le 01 mars 2021, consulté le 18 septembre 2021. URL : http://journals.openedition.org/cei/8832 ; DOI : https:// doi.org/10.4000/cei.8832

Ce document a été généré automatiquement le 18 septembre 2021. 


\title{
Autobiographies de la recomposition : esthétique d'une écriture conflictuelle dans l'œuvre de Barbara Balzerani
}

\author{
Autobiografie della ricomposizione: estetica di una scrittura conflittuale \\ nell'opera di Barbara Balzerani \\ Autobiographies of Recomposition: Aesthetics of Conflictual Writing in Barbara \\ Balzerani's Literary Work
}

Marie Thirion

Cette réflexion, partant de l'analyse de l'œuvre littéraire de Barbara Balzerani, vise à mettre en lumière la façon dont elle a articulé une double activité de militante politique et d'écrivaine. Les qualités littéraires et la spécificité de son écriture, soulignées par de nombreux critiques $^{1}$, rencontrent cependant encore un certain silence de la part du monde académique et de l'analyse littéraire. Les textes de Balzerani - surtout le premier - comme d'autres "autobiographies armées ${ }^{2}$ », ont en effet davantage suscité l'attention des historiens et des journalistes qui, en insistant presque exclusivement sur la question de la violence politique, ont interrogé la possibilité d'utiliser ces textes comme sources fiables - ou non - pour l'historiographie ${ }^{3}$, ou ont cherché à catégoriser ces différentes productions des militants d'extrême gauche ${ }^{4}$. Je voudrais ici focaliser mon attention sur la dimension littéraire de l'œuvre de Balzerani. Il ne s'agit pas d'oublier qui écrit, ni de mettre de côté le caractère politique d'un texte pétri de conflictualité, mais plutôt d'interroger l'esthétique des œuvres d'une auteure qui a effectivement aussi été une militante des Brigades rouges.

2 Née à Colleferro, une ville-usine du Latium en 1949, Barbara Balzerani grandit dans une famille ouvrière et elle a 19 ans en 1968, lorsque les révoltes étudiantes s'intensifient dans la péninsule. C'est à ce moment qu'elle part étudier la philosophie à Rome où elle 
rejoint les mouvements ouvriers et étudiants ${ }^{5}$ de la capitale. Elle milite d'abord dans l'un des mouvements opéraïstes de la gauche extraparlementaire, Potere operaio, avant de rejoindre, en $1975^{6}$, les Brigades rouges ${ }^{7}$, le groupe armé révolutionnaire italien le plus connu depuis l'après-guerre. Elle occupe, au sein de l'organisation, un rôle de direction et de coordination des actions et prend part, en 1978, au commando qui enlève Aldo Moro, alors président de la Démocratie chrétienne et ancien président du Conseil de la République italienne. Après son entrée dans la clandestinité en 1978, elle dirige à partir de 1981 les Brigades rouges-Parti communiste combattant (BR-PCC), faction née de la scission de l'organisation, jusqu'à son arrestation en 1985. Elle est condamnée à la perpétuité lors du procès "Moro-ter» et jugée avec d'autres brigadistes comme responsable de l'assassinat de Moro. Avant sa libération définitive en 2011, elle a passé 26 ans en prison, où elle se diplôme en anthropologie et commence son travail d'écriture.

3 Je m'étais concentrée dans un premier temps sur Compagna luna, le premier texte écrit par Barbara Balzerani, proposant une hypothèse de lecture selon laquelle l'écriture représenterait une tentative, une méthode, un instrument de recomposition d'une identité et d'un parcours fragmentés, déchiquetés ${ }^{8}$. Il s'agissait plus précisément de comprendre avec quels moyens stylistiques Barbara Balzerani réussissait à donner un sens à son propre parcours politique en tissant une toile non seulement cohérente, mais aussi esthétiquement particulière. C'est d'ailleurs dans ce texte que Balzerani évoque le plus son expérience de la lutte armée en tentant davantage d'en raconter les raisons, les conséquences personnelles et collectives et surtout les interrogations qui en résultent, plutôt que de justifier ses choix politiques et de chercher l'absolution. Les ouvrages suivants, sans taire cette expérience, disent bien d'autres histoires. Dans La sirena delle cinque ${ }^{9}$, écrit, comme le premier ouvrage, durant l'incarcération, Balzerani se concentre sur son enfance, sa famille, le travail à l'usine de ses parents et sur les années passées en prison. Les deux livres suivants sont rédigés, quant à eux, pendant la période de liberté conditionnelle de l'auteure. Perché io, perché non tu $\mathbf{u}^{10}$ revient lui aussi, dans les premières pages, sur son enfance jusqu'aux manifestations romaines de la fin des années soixante, mais est surtout dédié au récit de l'expérience carcérale de Balzerani jusqu'aux années de la liberté conditionnelle et de la découverte des transformations d'une Italie ensanglantée par le G8 de Gênes et par les souffrances des migrants « extra-communautaires ", nouveaux «boucs émissaires ${ }^{11}$ » du deuxième millénaire. Cronaca di un'attesa ${ }^{12}$ raconte la dernière année précédant la libération définitive de l'auteure. Elle narre une fois encore les ravages industriels de son village d'enfance mais surtout les scandales contemporains, de l'assassinat de Stefano Cucchi aux migrants noyés et ignorés de la Méditerranée, des transformations de la ville postmoderne à la misère des Roms de Milan. Les derniers ouvrages du corpus sont enfin rédigés après la libération définitive. Dans le cinquième livre, Lascia che il mare entri ${ }^{13}$, elle écrit sur trois générations de femmes, mêlant les existences de son arrière-grandmère, de sa mère et la sienne, et rapporte leurs dialogues réels ou imaginaires. Son dernier ouvrage à ce jour, $L$ 'ho sempre saputo ${ }^{14}$, élargit encore le champ des réflexions et des thématiques de l'auteure. Elle met en effet en scène un dialogue onirique entre le « je » de la militante incarcérée et une femme africaine effectivement rencontrée en prison, dialogue au sein duquel sont enchâssées d'autres voix qui interrogent le monde précédant la domination occidentale et les conséquences de cette dernière. l'ensemble de l'œuvre de l'auteure ${ }^{15}$, dont la teinte résolument autobiographique ne 
peut en aucun cas être réduite au seul récit de l'expérience de la lutte armée. Ainsi, ce travail pourrait constituer une première borne pour l'analyse littéraire de ces textes, parcourus de nombreux thèmes tels que la nature, la prison, le corps, la ville, que je n'ai $\mathrm{pu}$, faute de temps et de place, approfondir dans ce cadre.

\section{Les lacérations de la mémoire et de l'identité}

5 Deux motivations semblent sous-tendre l'écriture de Barbara Balzerani. La première, évoquée dans l'incipit de son premier ouvrage, est celle de vouloir rompre «un assordante silenzio in cui è interdetta la memoria e la libera parola ${ }^{16} »$ des militants d'extrême gauche, « vinti ${ }^{17}$ » et souvent incarcérés, silence sur lequel nous reviendrons. La seconde est exprimée à travers une image, celle de la lacération, du morcellement de l'histoire des années de lutte et de sa propre identité. Dans les notes qui ouvrent la réédition de son premier livre en 2013, cet espace paratextuel où elle revient, quinze ans après la première édition, sur le sens de son texte, Balzerani affirme en effet que « queste pagine sono il racconto dell'inizio di un viaggio di ritorno tra le schegge di uno specchio andato a pezzi, riflessi di una vita frantumata ${ }^{18} »$. Outre la métaphore du voyage, souvent convoquée dans ses textes, elle utilise ainsi celle d'un miroir brisé reflétant une vie elle aussi morcelée. Cette fragmentation n'est pas seulement individuelle, elle est aussi celle d'une génération de militants vaincus, comme l'auteure le suggère quelques lignes plus bas : «Avevo tra le mani una storia ridotta a brandelli, piegata alla lettura della resa dei conti dopo la rivolta degli anni Settanta ${ }^{19}$.»

6 Le topos de la fragmentation parcourt le genre autobiographique, surtout avec l'abandon de l'illusion d'une certaine unité du «je » favorisant, depuis le début du $\mathrm{xx}^{\mathrm{e}}$ siècle, la représentation d'une "personne divisée ${ }^{20}$ " plus en adéquation avec la complexité du sujet notamment mise en lumière par la psychanalyse ${ }^{21}$. La dispersion du « moi » de la modernité et la reconnaissance de la dimension sélective de la mémoire ont par ailleurs influencé les formes même de l'auto-narration où le "fragment » apparaitt privilégié de manière récurrente ${ }^{22}$.

7 Balzerani parle bien de « frammenti di vita costretti in ingorghi di emozioni [che] si animano in nuove composizioni per l'interferenza di un'eco, il frammento di un'immagine $^{23}$ " ou des «incastri del caleidoscopio in cui sta girando la [sua] vita ${ }^{24}$ " pour suggérer le morcellement des souvenirs et les difficultés du travail de la mémoire. Mais lorsqu'elle fait allusion aux conséquences de l'engagement politique des militants des Brigades rouges et plus généralement du mouvement des années soixante-dix, les termes sont plus incisifs, plus violents. Dans les passages de l'incipit précédemment cités, l'histoire personnelle et collective des militants n'est en effet pas seulement divisée et fragmentée mais " frantumata ", « ridotta a brandelli ${ }^{25}$ ", et c'est l'image d'un paysage dévasté, réduit à « macerie » et « rovine ${ }^{26}$ ", qu'elle convoque encore dans son dernier ouvrage pour représenter leur « sconfitta».

8 Le récit de son propre parcours semble par ailleurs lui aussi procéder «a strappi ${ }^{27}$ ». Dans Compagna luna, le texte qui propose la structure la plus linéaire et qui correspond le plus au genre autobiographique tel qu'il a été défini par Philippe Lejeune ${ }^{28}$, l'enfance de l'auteure est d'abord évoquée comme une sorte d'âge d'or :

I prati e la grande libertà di giornate a sfidare i limiti della realtà nelle più immaginifiche esplorazioni. El'avventura quotidiana a sopperire generosamente alla mancanza di favole, giocattoli e presenze. [...] Ma ne poteva fare a meno. 
A compensare, un instancabile ripercorrere su e giù gli spazi di quel piccolo immenso mondo che regalava l'appagamento di reinvenzioni e scoperte continue, di insospettabili tesori e opportunità, fino a che sosteneva la fantasia. Ogni giorno da capo $^{29}$.

9 L'imagination, la fantaisie et la nature semblent ainsi avoir permis à la narratrice de combler les "privazioni materiali ${ }^{30}$ » et affectives d'une époque où domine un temps circulaire marqué par les réitérations. Mais la découverte, lors de son entrée à l'école, des inégalités du «mondo degli adulti, quello in cui vigono le differenze e in cui alla conoscenza si paga lo scotto del giudizio e della competizione ${ }^{31}$ » apparait comme une première rupture. Ainsi, comme elle l'affirme quelques pages plus tard, « il tarlo aveva scavato e penetrato fino a farle perdere ogni sapere di perdute armonie ${ }^{32}$ ", évoquant par un polyptote cette double perte de l'insouciance de l'enfance. Cette première déchirure involontaire est suivie par d'autres. Son départ à Rome pour participer aux occupations universitaires et aux manifestations de 1968 est par exemple décrit comme "senza [...] ritorno ${ }^{33}$ ", comme une manière de "tagli[are] i ponti ${ }^{34}$ " avec sa vie précédente, de «strappare, strappare da sé ogni legame, [...] fare a mille pezzi ogni ricordo e rinascere per smentire e provare che tutto poteva essere diverso ${ }^{35}$ ". L'enthousiasme de la révolte contre l'humiliation et les divisions sociales et le système patriarcal ${ }^{36}{ }^{\prime}$ 'apparente donc à une nouvelle naissance qui ne peut s'accomplir sans la destruction de son existence précédente, sans cette « radicalità di scelte ${ }^{37}$ » qui marque le militantisme de l'auteure. De même, après son entrée dans les Brigades rouges, son extranéité avec les mouvements féministes dont elle critique notamment « l'accent mis sur la division en genres et non en classes ${ }^{38}$ ", est qualifiée de "strappo", de "separazione ${ }^{39}$ ", de "distacco $0^{40}$ ». Ce détachement est par ailleurs réciproque et permet à l'auteure de problématiser non sans ironie, évoquant une polémique à son propos survenue après son arrestation, l'incompréhension médiatique et sociale suscitée par le choix effectué par des femmes de participer à la lutte armée : «[...] non c'era infatti nulla da capire. Tutto era già compreso nelle scelte violente che avevano scavato un baratro tra lei, la terrorista, e il resto del genere femminile ${ }^{41}$. »

Le récit des déchirures biographiques, dont la portée politique est évidente, jusqu'aux lambeaux de la défaite, illustre bien cette "vita frantumata " de l'auteure qui, se défendant de vouloir faire une "storia delle Brigate rosse " et affirmant ne raconter qu' " una parte di quanto $h[\mathrm{a}]$ vissuto e di come ", confère toutefois une dimension collective à son propos le qualifiant de «tentativo di riconnettere una storia collettiva attraverso le diverse stagioni di un'esistenza ${ }^{42}$ ». Et c'est à travers l'écriture, « fissando e ordinando nella materialità dei segni ${ }^{43}$ » que cette recomposition peut avoir lieu.

\section{L'écriture comme recomposition}

Si le genre autobiographique apparaît comme la tentative de donner un sens à sa propre existence à travers une reconstruction rétrospective agençant les mots et les événements racontés, les œuvres de Balzerani donnent à voir, plus qu'une reconstruction, une véritable recomposition d'une identité et d'une histoire, à la fois personnelle et collective. Ce projet est à de nombreuses reprises explicité par l'auteure, en particulier à travers la métaphore du tissage.

12 L'activité du tissage, ou plutôt celle de la couture, est d'abord représentée dans les textes. Apanage féminin, cette représentation n'apparaît pas sans ambiguïté dans le 
regard de la narratrice. Son admiration pour la fabrication de l'habit nuptial de sa sœur " uscito dalle mani di creature celesti ${ }^{44}$ » ou pour ces " miracoli di richiamo in vita di indumenti e biancheria dalla sfinitezza " opérés par les "regine del recupero ${ }^{45}$ " est évidente. Et pourtant, on sent aussi, médié par la subjectivité de sa mère ${ }^{46}$, le refus d'être emprisonnée dans cette division des genres, de "rincretinirsi con aghi e ferri ", la couture lui semblant «il triste riassunto della decantata pazienza delle mani di donna $^{47} »$.

Outre cette représentation, la référence au tissage, plus qu'à la couture, en ce qu'elle correspond à la fabrication du matériau, semble évoquer la conception même de l'écriture de l'auteure qui l'associe souvent à l'image du voyage ou à l'effort de remémoration. Dans La sirena delle cinque, les « promenades » dans la cour de la prison, «avanti e indietro con la smania di trattenere ancora vita intrecciando racconti di viaggio $^{48}$ ", rappellent la navette du métier à tisser, tandis que Balzerani évoque, quelques chapitres plus tard la «ricerca dei fili sulle cui tracce mettev[a] passi per ricomprendere la [su] a esistenza nell'ultima sua versione ${ }^{49}$ \%. Dans Perché io, perché non tu, c'est encore durant un voyage, celui vers une prison du nord de l'Italie après la sortie de l'isolement, qu'elle cherche à «sciogliere l'uno dall'altro i fili che tengono legati gli incastri del caleidoscopio in cui sta girando la [su]a vita ${ }^{50}$ ». Puis dans le chapitre évoquant l'enlèvement de Moro, elle affirme: «Debbo riallacciare molti fili per rientrare in quella visione della politica, in quella determinazione, in quel contesto ${ }^{51}$. » Dans Lascia che il mare entri, elle fait le récit de ces "tre generazioni di donne per riallacciare il filo delle [su]e origini incerte ${ }^{52}$ ». Enfin, sa dernière œuvre propose elle aussi plusieurs références à cet art, là encore en l'associant, à propos du récit de la femme africaine, au voyage ${ }^{53}$, au travail de la mémoire ${ }^{54}$ et finalement au partage, à la collaboration politique décrite cette fois avec une comparaison, « come i fili che tessono la trama di inediti percorsi di liberazione in un unico disegno $0^{55} »$. Les liens entre l'écriture et le tissage remontent au moins à l'Antiquité laissant des traces dans de nombreux mots ou expressions du langage usuel ${ }^{56}$. Au-delà de ce topos, Balzerani évoque une référence plus précise quant à cette métaphore :

Pour moi l'écriture a été et est cela: elle est une confidente, elle a une fonction thérapeutique et même réparatrice. Elle est tels ces fils que tisse l'artiste Maria Lai avec une grande maîtrise, elle contient d'antiques déchirures et lacérations, encore douloureusement ouvertes ${ }^{57}$.

Puisant son inspiration dans l'œuvre de l'artiste sarde Maria Lai, qui sut transformer des métiers à tisser en objets d'art et fabriquer des livres cousus où les fils de coton forment des mots, Balzerani confère ainsi des vertus réparatrices à une écriture qui ne masque toutefois pas ces "antiques déchirures et lacérations " mais en laisse voir les sutures, et ce à travers différents procédés.

D'abord, la tentative de Balzerani de recomposer son identité morcelée - entre femme et brigadiste, enfant et adulte, personne privée et personne publique - tout en donnant à voir cet effort passe à travers un choix typographique spécifique pour sa première œuvre. L'alternance de l'italique et du romain signale en effet l'alternance de deux points de vue. Dans le premier cas, la narratrice parle à la première personne, il s'agit d'une parole introspective, lyrique, émaillée de questionnements personnels. La graphie romaine témoigne quant à elle d'un positionnement plus distancié, souvent marqué par une focalisation externe à la troisième personne du singulier ou du pluriel et par un langage moins imagé, tenant parfois plus de l'essai politique que de la narration. Cette dichotomie, cette sorte de schizophrénie de la « "doppia" Balzerani ${ }^{58}$ " 
entre l'identité passée et présente, entre la personne privée et la personne publique, exprime bien ces lacérations du "moi» évoquées précédemment. Elle apparaitt par ailleurs comme un procédé de distanciation efficace favorisant l'introspection, la narration $\mathrm{du}$ « je » impliquant au moins un dédoublement temporel - entre les faits racontés et le moment de l'écriture -, sinon identitaire ${ }^{59}$. Cette alternance des points de vue permet enfin, si ce n'est une recomposition, du moins une juxtaposition, une coprésence des différentes facettes de la narratrice qui reconstitue ainsi, pour elle-même et pour ses lecteurs, une personne complexe, loin de l'image de la «terrorista dagli occhi di ghiaccio » en papier glacé véhiculée par la presse.

Outre les signes typographiques de cette tentative de recomposition identitaire, les traces du travail de mémoire, de la recomposition des souvenirs sont eux aussi visibles dans les textes de Balzerani. Dès Compagna luna, cet effort de remémoration est en effet narré et même mis en scène : «Per quanto mi sforzi proprio non ricordo. Non ricordo un solo momento dei miei primissimi anni. [...] Mi capita tra le mani una vecchia foto e, di colpo, ritrovo la bambina che sono stata ${ }^{60}$. » Elle souligne ainsi l'effort que requiert le souvenir et la nécessité d'un support matériel agissant comme déclencheur. Balzerani utilise d'ailleurs un procédé similaire dans Lascia che il mare entri où une lettre retrouvée, envoyée de Chicago par une cousine de sa mère, "rivela un capitolo inedito di una storia non vissuta che è anche la [su] $\mathrm{a}^{61} »$ déclenchant cette fois le travail non de la mémoire mais de l'imagination pour raconter les espoirs et les souffrances des migrants italiens en Amérique.

Le travail de remémoration est aussi représenté par les va-et-vient incessants de la narration entre différentes temporalités. Dans Compagna luna, ce procédé entaille à plusieurs reprises l'apparente linéarité structurelle du texte comme lorsque la description de l'effervescence politique romaine de la fin des années soixante est interrompue par le récit en italique du retour, "quasi in pellegrinaggio », de la narratrice au Campo dei fiori lors de son premier permis de sortie ${ }^{62}$. Les autres œuvres, moins linéaires, apparaissent souvent comme une série de photogrammes dont la succession recompose l'existence de la narratrice ou des êtres qu'elle évoque tout en en montrant les sutures. C'est le cas de Lascia che il mare entri où l'entrelacement des vies de l'arrièregrand-mère, de la mère de la narratrice et de cette dernière est par exemple assuré par l'emploi d'un vocabulaire tenant à l'expérience même de Balzerani que l'écrivaine applique à ses prédécesseuses. La situation des femmes au sein de la famille de sa mère est en effet définie comme "una prigionia senza colpa e senza riduzioni di pena ${ }^{63}$ " tandis que la narratrice exprime sa surprise en narrant la « decisione autonoma e di segno contrario » de sa mère de voter contre les indications de son père étant donnée la «clandestinità in cui viveva ogni giudizio critico $^{64}$ ». Dans L'ho sempre saputo, c'est en revanche l'anaphore " ricordo ${ }^{65}$ » qui, dans le premier chapitre, apparaît comme une sorte de point d'arrêt signalant le passage d'un souvenir à l'autre.

17 Cet effet de tissage ne se limite pas aux œuvres prises singulièrement mais apparait aussi entre les textes qui composent le corpus, à travers un dispositif d'échos qui en assure la cohérence. Certains épisodes de la vie de la narratrice sont ainsi évoqués à plusieurs reprises d'un texte à l'autre comme le début de son militantisme au sein des occupations et des manifestations du tournant de la décennie ${ }^{66}$ ou bien la mort de Fabrizio, un jeune homme de vingt ans, tué pendant les luttes en défense des «case occupate » d'un quartier de la périphérie de Rome, mort d'abord évoquée dans Perché io, perché non tu ${ }^{67}$ puis racontée dans Cronaca di un'attesa ${ }^{68}$. Les liens entre les œuvres 
peuvent encore être établis par la récurrence d'un thème comme celui de la mer qui ouvre Cronaca di un'attesa et conclut Lascia che il mare entri ou bien par des sortes d'annonces programmatiques peut-être inconscientes comme c'est le cas dans Compagna luna où, dans le chapitre «Una rivoluzionaria di professione », la narratrice énonce en italique :

Mi scorrono davanti tutte, le donne della mia famiglia. Almeno tre generazioni. E riconosco $i$ segni di una genealogia che fa la loro diversità tutta discendente da colei che ha messo in moto la sequenza. Quanto ancora perché l'indicibile di una ritrovata alleanza possa essere messo in parola ${ }^{69}$ ?

Il faudra attendre seize ans pour que Balzerani narre cette « ritrovata alleanza » entre ces trois générations de femmes dans Lascia che il mare entri.

Ces effets d'échos, loin d'être statiques, révèlent enfin la progression des réflexions politiques et existentielles de l'auteure. Dans Compagna luna, si une critique de la foi dans le progrès n'apparaît qu'esquissée ${ }^{70}$, Balzerani développe et approfondit ce thème dans L'ho sempre saputo, reconnaissant peut-être dans cette illusion l'une des principales erreurs politiques des Brigades rouges et d'une génération entière de militants :

[...] noi eravamo la generazione che chiamava genocidio la scoperta dell'America e consideravamo la vittoria di Little Big Horn come nostra. Ma neanche per un minuto ci veniva in mente che il modo di vivere degli indiani fosse paragonabile alle meraviglie dello sviluppo delle nostre forze produttive che, come l'Atala rossa di Stalin, ci avrebbero portato direttamente al comunismo, senza bisogno di nessuna transizione. Modello universale, buono per tutte le latitudini. Eravamo strabici, nell'incapacità di leggere il percorso altrui dentro il farsi di una storia e non secondo la nostra scala di valori. Una verità si fa strada. Non essere mai stati dalla parte dei conquistatori non ci ha salvati dalla loro influenza e ci siamo sentiti determinanti nello scontro per la libertà e l'eguaglianza di tutti. Non ci hanno sconfitti i padroni. In certa misura, siamo stati noi i padroni ${ }^{71}$.

19 Certainement influencée par ses études d'anthropologie et par le temps de la réflexion, Balzerani réitère cette autocritique lors de la présentation de son dernier ouvrage à Florence en mars 2018 : elle reconnaît leur aveuglement et qualifie d'" errore storico " le fait d'avoir cru à « l'idea di progresso, [...] di una storia progressiva che si lascia alle spalle tutto quello che non è all'altezza del momento ${ }^{72}$ ».

\section{Le pouvoir vital de la voix}

Cette remise en cause de la supériorité occidentale s'accompagne, dans l'œuvre de Balzerani de la nécessité de raconter "une autre histoire ${ }^{73}$ ", celle des classes subalternes, " cambiando il punto di osservazione ${ }^{74}$ » et en restituant la parole à ceux qui en ont été privés. En effet, outre les lacérations de l'histoire et de l'identité, Balzerani évoque, comme moteur de son écriture, le besoin de rompre « un assordante silenzio in cui è interdetta la memoria e la libera parola ${ }^{75}$ ». Ce silence est d'abord celui, collectif, des militants après la fin de la saison des mouvements qui a correspondu, pour beaucoup, à l'incarcération, à la réclusion dans un espace d'où la parole peine à sortir et à être entendue. Balzerani insiste sur ce silence et réaffirme, dans les notes qui ouvrent la réédition de l'ouvrage en 2013 , le prix payé selon elle pour cette "défaite » des militants d'extrême gauche, cette " pattuglia di scampati, zittiti dal coro cortigiano del vincitore $^{76} »$ : « Come pena accessoria per i vinti, la parola negata. Condanna non scritta a una galera impenetrabile. È capace di rimanere in gola a strozzare quella parola, dopo aver trovato alimento in ogni goccia di sangue, ogni piega di carne ${ }^{77}$. » Ce silence est 
aussi le sien, réduite comme elle l'était à un "muto parlare fra [sé] e [sé] ${ }^{78}$ " dans la cellule de prison, sur lequel elle revient par une sorte de jeu de miroir lorsqu'elle semble enfin réussir à se reconnaître, ou du moins à éprouver de l'empathie, pour la brigadiste représentée dans le film La seconda volta de Mimmo Calopresti :

Comincia a sembrarmi secondario il senso di fastidio per l'afasia della donna. Non può parlare, forse non perché non sa, ma perché non deve. Non è previsto che abbia facoltà di parola e anche quando l'avesse le va tolta. Perché? Perché la vendetta sociale per la sua colpa è la condanna al silenzio, tanta per lei l'illegittimità di usare una parola sensata che possa rendere ragione all'irragionevole. Non deve parlare. Può solo essere parlata e fornire un'immagine accomodante a lenimento dell'inquietudine che promana da una memoria troppo devastata per acconsentire l'inequivocabilità di una perdurante rassicurazione ${ }^{79}$.

C'est une injonction au silence que Balzerani a d'ailleurs subi de la part d'Antonio Tabucchi ${ }^{80}$ qui, après la publication de son premier livre, «con una terza pagina nazionale ha tolto a [lei] il diritto di parola ${ }^{81} »$ en imposant à la maison d'édition Feltrinelli de choisir entre lui et elle.

Mais ce silence est aussi, plus généralement, celui des « vaincus » de l'histoire qui n'ont pas leur place dans la «storia ufficiale, quella che usa le parole solo dopo averle disincarnate, nel linguaggio recintato del dominio che soffoca il respiro alla vita e alla liberazione ${ }^{82}$ ". Ainsi, l'œuvre de Balzerani peut être lue comme la tentative de restituer la parole aux subalternes en narrant d'autres histoires. Dans le chapitre de La sirena delle cinque consacré à son frère, elle affirme ainsi que

[...] la sua è una piccola storia. Ma le piccole storie, quelle che vivono le persone in carne e ossa quasi sempre sono la smentita della storia ufficiale e da questa ignorate. Eppure la loro narrazione ne è la figurazione più significante, la metafora che ne racchiude il senso e ne restituisce il segno partigiano. L'unico che può ristabilire intelligenza degli avvenimenti che contano nell'esistenza di ciascuno ${ }^{83}$.

Elle montre ainsi la nécessité de l'incarnation de l'histoire qui permet de lui donner un sens politique, "partigiano ». Outre celle de son frère, elle raconte aussi l'histoire des générations de femmes qui l'ont précédée dont sa mère qui «non aveva voce, come la maggioranza dei senza poteri come lei ${ }^{84}$ ", celle de son oncle "ecologista " dont le " presentimento che non riusciva neanche a formulare » sur l'empoisonnement de l'eau de la rivière de Colleferro s'oppose à la « rappresentazione iconografica ufficiale ${ }^{85}$ » ou encore celle des habitants victimes de la catastrophe du Vajont ${ }^{86}$, «senza voce per $\mathrm{i}$ decenni a venire e senza attestati accademici ma portatori di un sapere che a tecnocrati, politici e affaristi non parla mai, come fosse una lingua intraducibile ${ }^{87}$ ».

S'inspirant peut-être des réflexions de certains anthropologues sur le dialogisme ${ }^{88}$, Balzerani cherche non seulement à restituer ces voix subalternes mais aussi à les faire entendre, à les faire résonner dans ses textes, et ce, une fois encore avec l'expédient de la typographie. Dans La sirena delle cinque, les voix de membres de la famille de la narratrice scandent ainsi le texte, souvent à travers une parole oralisée comme dans le chapitre "Tifo ", où l'oncle Romolo est mis en scène saluant son héros - «Garibà vuoi beve? No?! Allora bevo io »- ou bien commentant un match de foot - «Ma quanto ci mettono 'sti cretini in mutande a sfondare 'sta palla ${ }^{89}$ ?». Ces voix sont aussi des réminiscences de paroles dont le locuteur n'est pas précisé avec lesquelles dialogue la narratrice ${ }^{90}$ ou qui prennent la forme de "formul[e] scaramantic[he] ${ }^{91}$ " comme la question «Che vuoi fare da grande?", titre du premier chapitre de Perché io, perché non tu, qui rythme les premières pages de l'ouvrage ${ }^{92}$. 
24 La polyphonie des textes de Balzerani cohabite enfin avec une mise en relief de la transmission de la parole, capable de perpétuer ces existences oubliées par l'histoire officielle. La narratrice est dans ce cas l'instance chargée de cette transmission comme en témoignent certains passages méta-discursifs des œuvres. Dans Cronaca di un'attesa, le deuxième chapitre s'ouvre ainsi sur une réflexion à propos de l'abîme qui se creuse entre ce que la narratrice apprend à l'école de la révolution industrielle et la réalité du travail de sa mère :

[...] si sa, un conto sono i processi storici, tutto un altro le vite delle persone. Specie quelle che se li caricano sulle spalle e arrivano per ultime a riscuoterne qualche vantaggio, quando ci arrivano. Per questo esistono i libri e quelli che hanno studiato a spiegare come è andata. E se la percezione non coincide, la colpa è del punto di osservazione di chi è chino sul particolare, quasi sempre parziale e deviante. Adesso mia madre non c'è più. $\mathrm{E}$ mi manca per ogni cosa. Lei che mi avrebbe aiutato a farne un altro racconto. Di quelli che si trovano in pochi libri. Ci provo, caricandomi la sua fatica sulle spalle. Comincio a vedere ${ }^{93}$.

Ce procédé de transmission de la parole apparaît de manière encore plus claire dans L'ho sempre saputo, où l'enchâssement des récits structure le texte même. L'histoire de la planète précédant les ravages de la domination occidentale et la narration de ses conséquences ont été racontées à la femme africaine par son dernier enfant lorsqu'elle l'avait encore dans son ventre. Elle les raconte à son tour à la narratrice, chargée de «far prendere forma ai messaggi che il suo ventre aveva contenuto ${ }^{94}$ » pour les transmettre au lecteur à travers le processus maïeutique de l'écriture. Ce choix de l'enchâssement, qui donne une dimension presque mythique au récit, témoigne peutêtre aussi de la volonté - politique - de l'auteure de s'inscrire dans une tradition narrative issue surtout de l'héritage oriental ${ }^{95}$ afin de critiquer l'Occident.

Car cette opération de restitution de la voix s'accompagne bien d'une réflexion sur la dimension politique de toute écriture de l'histoire et sur le langage même utilisé pour dire le monde et les événements humains. Dans Perché io, perché non tu, Balzerani affirme ainsi :

Il novecento ha portato via con sé anche il vocabolario e la simbologia con cui aveva costruito le sue narrazioni. [...] La memoria non offre categorie interpretative sufficienti a dare ragione dell'inclinazione diversa in cui si è messo a girare il pianeta. [...] Così la guerra è diventata umanitaria, la razzia di materie prime sostegno all'altrui democrazia, i reticolati garanzia di sicurezza sociale, la negazione delle libertà personali difesa della moralità condivisa, con qualche onorevole eccezione ${ }^{96}$.

27 À travers cette énumération illustrant l'usage actuel de la « langue de bois ${ }^{97}$ ", basée sur une antinomie visant à voiler sinon à cacher le réel, Balzerani insiste sur l'inadéquation du langage du siècle passé pour dire le présent, un présent à son tour phagocyté par la technologie qui menace les facultés mêmes de la pensée, de la communication et de la mémoire comme elle l'énonce dans L'ho sempre saputo :

[...] mentre comunichiamo con un mondo di immagini disimpariamo le parole e impoveriamo il pensiero. [...] la tecnica, all'esclusivo servizio dell'interesse privato, non sta sostituendo più solo l'intelligenza di millenni di sapere ma sta colonizzando la stessa capacità di comunicare, conoscere e scegliere, piantandoci un microchip nel cervello. In un presente storico digitale che ha inglobato e confuso ogni altro tempo, in cui nulla inizia e si conclude e la memoria non è esperienza tramandata ma lo spazio su un disco. In cui siamo contenitori di informazioni che non elaboriamo perché il tempo non è più quello umano ma quello di un processore ${ }^{98}$. 
Balzerani, le langage a donc une forte portée politique et son écriture prend place dans un « conflitto semantico ${ }^{99}$ " où la parole semble indissociable de la lutte et de la vie.

En effet, au sein de ces structures textuelles s'apparentant à des poupées gigognes, l'acte narratif est souvent mis en scène notamment en ce qui concerne les récits de son père et de sa mère ${ }^{100}$. Ceux-ci semblent d'abord avoir un pouvoir d'évocation si puissant qu'ils permettent de façonner le réel. La narratrice affirme ainsi dans Cronaca di un'attesa:

[...] [le] malie di mio padre [...] mi consentivano di continuare a far prendere al mondo la forma delle sue narrazioni. Perché lui conosceva luoghi all'altezza dei giardini delle delizie che mi descriveva mettendoci dentro anche i personaggi famosi che li frequentavano e, sempre, le cose buone che lì si mangiavano. E noi un giorno ci saremmo andati ${ }^{101}$.

Ces histoires racontées lorsque la narratrice était enfant lui procurent en outre un certain réconfort. Ainsi, dans Perché io, perché non tu, elle évoque la cuisine, où « padre $\mathrm{e}$ madre narravano storie vissute e di generazioni passate come un'epopea ", qui était "l'unico posto di tutta la casa dove faceva caldo. Per la stufa e per i racconti ${ }^{102}$ ». De même, dans La sirena delle cinque, le souvenir de ce même lieu revient, celui où son père " cantava romanze » et « in quelle occasioni il sugo per la pasta era più generoso del solito ma ancora più intenso il gusto dei suoi racconti ${ }^{103}$ ». Les récits du père ne se contentent pas de sublimer le réel mais offrent aussi une échappatoire à la misère comme en témoignent les mots de la narratrice : «Lui mi teneva legata al filo del suo narrare e come potevo, in quell'incantamento, accorgermi del pavimento crepato e delle macchie d'umidità agli angoli del soffitto annerito ${ }^{104}$ ? " Ce pouvoir de transfiguration du réel apparaît encore dans L'ho sempre saputo, lorsque le récit de la femme africaine se montre capable d'éliminer la condition carcérale dans laquelle se trouvent les deux prisonnières : «La cella disadorna che ospitava i nostri incontri non era più quella di prima. Come fosse diventata più grande. Senza mura, né sbarre, né guardie. Miracolo del contagio di energia liberatoria della mia compagna di viaggio ${ }^{105}$. » L'« incantamento ", le « miracolo » du récit confèrent ainsi une dimension libératoire au verbe qui prend pour la narratrice un caractère vital, existentiel. Dans le même texte, évoquant encore la capacité du récit de la femme de faire disparaître les murs de la cellule, elle décrit les effets que cette parole lui provoque :

Lei parlava e lo spazio della cella si dilatava a misura di un'inondazione di luce che non trovava argini e non trascinava via, ma inondava benefica, fin le nostre viscere di donne. Fino a sentirsene parte. Un frammento di me rinasceva a nuova vita nei solchi lasciati dalla spina della sua nostalgia. Travaso di energia vitale di un mondo scomparso e richiamato in vita tra gli squarci di una memoria dei sensi, colori, fiuti, indizi e contagio di ragione vitale a nutrire il racconto ${ }^{106}$.

Dans ce passage, l'isolexisme portant sur le substantif «vita » dont dérive l'adjectif « vitale », répétés chacun à deux reprises, tout comme l'antanaclase du mot « vitale » (relatif à la vie mais aussi, au sens figuré, " essentiel, très important ») et l'utilisation du substantif dans deux expressions évoquant la renaissance (« rinascere a nuova vita » et « richiamare in vita»), la sienne et celle du monde, mettent en relief la dimension existentielle que la narratrice attribue au récit.

Par un jeu d'associations réticulaires parcourant les œuvres, Balzerani crée alors une sorte de triangulation entre la voix, la vie et la lutte. Dans le premier chapitre de L'ho sempre saputo dans lequel elle revient sur les révoltes de la fin des années soixante, 
l'association entre vie et lutte est établie par le biais d'un chiasme résonnant à distance de quelques pages. En effet, la narratrice évoque d'abord la nécessité de « riappropriarsi del senso della vita. E della lotta ${ }^{107}$ ", puis, pour conclure le chapitre, celle de « trovare altre strade per continuare a lottare. $\mathrm{E}$ a vivere ${ }^{108}$ ». Le parallélisme de la construction, ainsi que la variation morphologique créent une résonnance entre les deux passages qui met en lumière le caractère indissociable de ces deux termes. Dans Cronaca di un'attesa, à propos des transformations de l'Italie et du manque de conflictualité politique, la narratrice construit un lien supplémentaire entre voix, vie et lutte en affirmant: "La partita in gioco è la vita e la voce di un bel pezzo di questo paese che oggi resiste in solitudine ${ }^{109}$. Ce lien est réitéré dans Perché io, perché non tu, lorsque, à la suite du passage concernant la langue de bois de la communication politique actuelle, elle indique quelles formes prennent les luttes des nouveaux mouvements sociaux :

Il potere ha perfezionato la sua pervasività in ogni campo del vivere e, come sempre, l'antinomia su cui poggia genera anche la sua negazione. Per questo i riferimenti ideali dei nuovi movimenti parlano la lingua della vita, di realizzazioni presenti che vanno al ritmo della velocità dei sogni $[. . .]^{110}$.

$\mathrm{Au}$ terme de notre étude, l'écriture apparaît donc comme un instrument de recomposition du miroir brisé, métaphore de la vie de l'auteure, mais aussi de l'histoire d'un siècle, des raisons de la conflictualité politique. Loin du soliloque, les textes de Balzerani, faits de "parole sporcate di vita vissuta ${ }^{111}$ ", prennent à leur tour la forme d'un miroir tendu au lecteur qui ne peut qu'interroger son propre être au monde et les raisons des lacérations conflictuelles de ce monde.

\section{NOTES}

1. Voir notamment le florilège sélectionné par Barbara Balzerani dans la « Nota dell'autrice alla nuova edizione" de Compagna luna, Rome, DeriveApprodi, 2015 (1998), p. 6-10. Le texte a récemment été traduit en français: B. Balzerani, Camarade Lune, traduction de M. Baccelli, Paris, éditions Cambourakis, 2019.

2. E. Betta, «Memorie in conflitto. Autobiografie della lotta armata », Contemporanea, vol. 12, $\mathrm{n}^{\circ} 4$, 2009, p. 676.

3. Voir notamment B. Armani, «La production historiographique, journalistique et mémorielle sur les années de plomb», dans M. Lazar et M.-A. Matard-Bonucci (éds), L'Italie des années de plomb : le terrorisme entre histoire et mémoire, Paris, Autrement, coll. " Mémoires/Histoire ", 2010, p. 192-207 ou, plus récemment, D. Bini, Donne e lotta armata (1970-1985), Rome, DeriveApprodi, 2017.

4. S. Wharmby, L'édition des témoignages de membres des Brigades rouges: manifestations du passé et vecteurs de mémoire, mémoire d'histoire dirigé par M.-A. Matard-Bonucci, Université Grenoble Alpes, 2007.

5. C'est notamment l'union de ces deux « âmes » qui caractérise le « mai rampant » italien, selon l'expression d'Emilio Reyneri (E. Reyneri, « Il maggio strisciante: l'inizio della mobilitazione operaia », dans A. Pizzorno (éd.), Lotta operaia e sindacato: il ciclo 1968-1972 in Italia, Bologne, il Mulino, 1978 p. 49-107), plus long et plus intense que dans d'autres pays comme la France. 
6. Selon Balzerani, le coup d'État au Chili de 1973 et l'enlèvement du juge Sossi par les Brigades rouges l'année suivante constituent les deux «événements décisifs » de son entrée dans la lutte armée (S. De Bernardinis, «Entrevista com Barbara Balzerani », Mouro, nº 10, 2016, p. 131-141 ; pour la traduction italienne, voir <https://beccodiferro.noblogs.org/barbara-balzerani-estraordinario-come-non-venga-formulato-nessun-interesse-sulle-reali-cause-della-nascita-e-delperdurare-della-lotta-armata/> [page consultée le 15 janvier 2020]). Elle affirme en effet que : «Il primo aveva rafforzato la convinzione che la via pacifica al socialismo era impossibile, che la democrazia formale era solo la faccia presentabile di un potere criminale e il secondo aveva mostrato che era alla portata di un'organizzazione di operai attaccare, giudicare e condannare lo Stato, come premessa per altre libertà necessarie e finalmente possibili. »

7. Parmi les nombreux ouvrages dédiés à l'histoire de l'organisation, on peut citer M. Clementi, Storia delle Brigate Rosse, Milan, Odratek, 2007, ainsi que M. Clementi, P. Persichetti et E. Santalena, Brigate rosse. Dalle fabbriche alla «campagna di primavera», Rome, DeriveApprodi, 2017.

8. Cette réflexion a pour origine l'intervention prononcée lors de la XVIII ${ }^{\mathrm{e}}$ édition du séminaire Work in Progress du LUHCIE, organisé à l'Université Grenoble Alpes, qui portait exclusivement sur le premier ouvrage de Balzerani.

9. B. Balzerani, La sirena delle cinque, Rome, DeriveApprodi, 2015 (2003).

10. B. Balzerani, Perché io, perché non tu, Rome, DeriveApprodi, 2009.

11. Ibid., p. 78.

12. B. Balzerani, Cronaca di un'attesa, Rome, DeriveApprodi, 2011.

13. B. Balzerani, Lascia che il mare entri, Rome, DeriveApprodi, 2014.

14. B. Balzerani, L'ho sempre saputo, Rome, DeriveApprodi, 2017.

15. Rédigée au début de 2020, la présente étude ne prend pas en compte le dernier ouvrage de Barbara Balzerani, Lettera a mio padre, publié chez DeriveApprodi en août 2020.

16. B. Balzerani, Compagna luna, ouvr. cité, p. 23.

17. Ibid., p. 6.

18. Ibid., p. 5.

19. Ibid.

20. R. Barthes, Roland Barthes par Roland Barthes, Paris, Seuil, 1995 (1975), p. 127.

21. P. Lejeune, Je est un autre : l'autobiographie, de la littérature aux médias, Paris, Seuil, 1980.

22. Voir par exemple S. Doubrovsky, Le livre brisé, Paris, Gallimard, 1989 ou A. Ernaux, « Fragments autour de Philippe V », L’Infini, n 56, décembre 1996, œuvres dont les titres mêmes annoncent la fragmentation.

23. B. Balzerani, La sirena delle cinque, ouvr. cité, p. 11.

24. B. Balzerani, Perché io, perché non tu, ouvr. cité, p. 37.

25. B. Balzerani, Compagna luna, ouvr. cité, p. 5.

26. B. Balzerani, L'ho sempre saputo, ouvr. cité, p. 27.

27. B. Balzerani, La sirena delle cinque, ouvr. cité, p. 11.

28. L'incipit a en effet tous les traits du "pacte autobiographique" tel qu'il est codifié par Lejeune : intentions de l'auteur, circonstances de l'écriture, définition - en creux - du lecteur et réponses à objections et critiques. Voir P. Lejeune, Le pacte autobiographique, Paris, Seuil, 1975.

29. B. Balzerani, Compagna luna, ouvr. cité, p. 27.

30. Ibid., p. 28.

31. Ibid.

32. Ibid., p. 34.

33. Ibid., p. 37.

34. Ibid., p. 36.

35. Ibid., p. 37.

36. Ibid.

37. Ibid., p. 36. 
38. B. Balzerani et M. Dell'Omodarme, « "Être une prolétaire est ce qui m'a poussée à adhérer à la lutte armée" : entretien avec Barbara Balzerani », traduction de Marco Dell'Omodarme, Comment s'en sortir?, no 4, printemps 2017, p. 46.

39. B. Balzerani, Compagna luna, ouvr. cité, p. 60.

40. Ibid., p. 61.

41. Ibid.

42. Ibid., p. 5 .

43. Ibid., p. 23.

44. B. Balzerani, La sirena delle cinque, ouvr. cité, p. 31.

45. B. Balzerani, Lascia che il mare entri, ouvr. cité, p. 53.

46. Dans ce chapitre, Balzerani évoque en effet l'enfance de sa mère, qui aurait aimé «fare parte della schiera dei maschi » et est en revanche reléguée aux travaux féminins (ibid.).

47. Ibid.

48. B. Balzerani, La sirena delle cinque, ouvr. cité, p. 14.

49. Ibid., p. 65.

50. B. Balzerani, Perché io, perché non tu, ouvr. cité, p. 37.

51. Ibid., p. 87.

52. B. Balzerani, Lascia che il mare entri, ouvr. cité, p. 6.

53. " Mi raccontò. Mi fece da guida in un viaggio che scosse le fondamenta di quello che pensavo di sapere [...] seguendo il ritmo dei fili della connessione collettiva che restituisce comprensione, come direbbe l'artista che ne ricama il disegno. " (B. Balzerani, L'ho sempre saputo, ouvr. cité, p. 29.)

54. «Non voleva perderne memoria e si aggrappava ai filamenti dei ricordi per ritesserne la trama. » (Ibid., p. 43)

55. Ibid., p. 61.

56. On peut penser à l'étymologie même du mot «texte ", « testo » qui vient du latin " texere », tisser, aux expressions françaises "la trame du discours », " de fil en aiguille », « un tissu de mensonges » et aux expressions italiennes "l'intreccio del libro ", « il filo della narrazione». Parmi les nombreuses études sur ce sujet, on peut consulter F. Rigotti, Il filo del pensiero. Tessere, scrivere, pensare, Bologne, il Mulino, 2002.

57. B. Balzerani et M. Dell'Omodarme, «Être une prolétaire », ouvr. cité, p. 50-51.

58. Pour reprendre le titre de la préface rédigée par Ivan Della Mea pour La sirena delle cinque, «I quadri della memoria della "doppia” Balzerani », dans B. Balzerani, La sirena delle cinque, ouvr. cité, p. 9.

59. P. Lejeune, Je est un autre, ouvr. cité ; S. Wharmby, L'édition des témoignages, ouvr. cité, p. 85.

60. B. Balzerani, Compagna luna, ouvr. cité, p. 25-26 (texte en italique original).

61. B. Balzerani, Lascia che il mare entri, ouvr. cité, p. 83.

62. B. Balzerani, Compagna luna, ouvr. cité, p. 40-41.

63. B. Balzerani, Lascia che il mare entri, ouvr. cité, p. 55.

64. Ibid., p. 56.

65. B. Balzerani, L'ho sempre saputo, ouvr. cité, p. 17, 19, 20 et 24.

66. Notamment B. Balzerani, Compagna luna, ouvr. cité, p. 40-42 ; Id., Perché io, perché non tu, ouvr. cité, p. 21-22 ; Id., L'ho sempre saputo, ouvr. cité, p. 15-28.

67. B. Balzerani, Perché io, perché non tu, ouvr. cité, p. 98.

68. B. Balzerani, Cronaca di un'attesa, ouvr. cité, p. 46-47.

69. B. Balzerani, Compagna luna, ouvr. cité, p. 119.

70. «Mentre il "bene in sé della produzione", travolgendo relazioni industriali inservibili nel dominio dell'impresa multinazionale, si attrezzava ad esuberare sempre più operai straparlando di un lavoro più pulito e sicuro - che oggi fa quattro morti al giorno fra i pochi sfortunati che si spartiscono un simile bene. » (B. Balzerani, Compagna luna, ouvr. cité, p. 57.) 
71. B. Balzerani, L'ho sempre saputo, ouvr. cité, p. 47.

72. "Questo credo che ci abbia abbagliato parecchio, [...] questo vecchio discorso, che ci ha accumunato un po' tutti, dello sviluppo delle forze produttive come viatico verso la costruzione di una società socialista di transizione verso il comunismo." Enregistrement audio du débat «Barbara Balzerani presenta il suo libro "L'ho sempre saputo" » organisé par le Centro popolare autogestito di Firenze, 16 mars 2018, disponible sur <www.radioradicale.it/scheda/536061/ barbara-balzerani-presenta-il-suo-libro-lho-sempre-saputo-ed-deriveapprodi> (page consultée le 19 janvier 2020).

73. D'après le titre de la présentation de Silvia De Bernardinis lors du festival DeriveApprodi qui s'est tenu au Nuovo Cinema Palazzo de Rome les 25, 26 et 27 novembre 2016, disponible sur <https://infodocks.files.wordpress.com/2016/10/un_altra-storia1.pdf> (page consultée le 19 janvier 2020).

74. B. Balzerani, L'ho sempre saputo, ouvr. cité, p. 48.

75. B. Balzerani, Compagna luna, ouvr. cité, p. 23.

76. Ibid., p. 5.

77. Ibid., p. 6.

78. Ibid., p. 23.

79. Ibid., p. 129.

80. A. Tabucchi, «Balzerani Compagna luna, fratello mitra », Il Corriere della sera, 5 juillet 1998. Dans cet article, Tabucchi affirme ainsi à propos du premier ouvrage de Balzerani : « È un fastfood di manualistica rivoluzionaria dove si danno la mano, a loro insaputa, un Lenin di propaganda ed un D'Annunzio di periferia, una mistica militare, reticenze, allusività, stereotipi, il linguaggio delle sentinelle del colonello Gheddafi ed un kitsch che ricorda i libretti di Henver Hoxha, i sentimenti di Sanremo e l'oggettistica dei santuari dove piangono le madonne. [...] Non si chiede a chi visse tali esperienze (e che soprattutto le fece vivere agli altri) che vent'anni dopo ne parli da Dostoevskij, o magari solo con l'ombra d'un dialettico dubbio. Ma chi decide di affrontare un simile argomento attraverso la scrittura deve avere il coraggio di scendere sino al nodo più profondo, sino al "cuore di tenebra". Se non ce l'ha, mantenga un decoroso silenzio, che è un'altra forma di coraggio."

81. B. Balzerani, Compagna luna, ouvr. cité, p. 10.

82. B. Balzerani, L'ho sempre saputo, ouvr. cité, p. 55.

83. B. Balzerani, La sirena delle cinque, ouvr. cité, p. 41.

84. B. Balzerani, Lascia che il mare entri, ouvr. cité, p. 56.

85. B. Balzerani, Cronaca di un'attesa, ouvr. cité, p. 29.

86. Voir à ce sujet le spectacle de Marco Paolini, « Il racconto del Vajont ».

87. B. Balzerani, Lascia che il mare entri, ouvr. cité, p. 79.

88. Voir notamment D. Tedlock, "Questions concerning Dialogical Anthropology », Journal of Anthropological Research, vol. 43, nº 4, p. 325-337, ainsi que J. Clifford et G. E. Marcus (éds), Writing culture. The Poetics and Politics of Ethnography, Berkeley, University of California Press, 1986. Une des inspirations de ce "tournant postmoderne" de l'anthropologie a notamment été la série d'articles de Mikhail Bakhtin publiés à titre posthume dans lesquels il introduit les concepts de « dialogisme » et d' « hétéroglossie » : voir M. Bakhtin, The Dialogic Imagination: Four Essays, Austin, University of Texas Press, 1994 (1981).

89. B. Balzerani, La sirena delle cinque, ouvr. cité, p. 43 (texte en italique original).

90. Comme dans ce passage où l'italique et la ponctuation semblent signaler l'écho de cette voix à laquelle répond ensuite la narratrice : « [...] Lasciateli andare i morti altrimenti non trovano pace [...] Ma il tempo a volte non è così galantuomo da accordare il distacco alla fretta del commiato [...]. » (B. Balzerani, Perché io, perché non tu, ouvr. cité, p. 36, texte en italique original.)

91. Ibid., p. 23.

92. Ibid., p. 13 et 23 . 
93. B. Balzerani, Cronaca di un'attesa, ouvr. cité, p. 25-26.

94. B. Balzerani, L'ho sempre saputo, ouvr. cité, p. 34.

95. Voir notamment T. Todorov, «Les hommes-récits », Poétique de la prose, Paris, Seuil, 1971, p. 78-91.

96. B. Balzerani, Perché io, perché non tu, ouvr. cité, p. 79-80.

97. Parmi les nombreux ouvrages consacrés à ce phénomène, on peut consulter J. Nowiki, M. Oustinogg et A.-M. Chartier, « Les langues de bois », Hermès, vol. 58, n 3, 2010.

98. B. Balzerani, L'ho sempre saputo, ouvr. cité, p. 26.

99. B. Balzerani, Cronaca di un'attesa, ouvr. cité, p. 33.

100. Les récits de la mère évoquent surtout les effets de la Première guerre mondiale sur la famille et le village natal comme dans Cronaca di un'attesa, p. 115, où la narratrice entend cette histoire pendant qu'elle marche, avec sa mère, «tra le sterpaglie », ou dans Lascia che il mare entri, où le récit prend cette fois une forme itérative : « Mi disse sempre di quella guerra [...]. » (p. 61)

101. B. Balzerani, Cronaca di un'attesa, ouvr. cité, p. 72.

102. B. Balzerani, Perché io, perché non tu, ouvr. cité, p. 40.

103. B. Balzerani, La sirena delle cinque, ouvr. cité, p. 26.

104. Ibid., p. 27.

105. B. Balzerani, L'ho sempre saputo, ouvr. cité, p. 43.

106. Ibid., p. 30.

107. Ibid., p. 23.

108. Ibid., p. 28.

109. B. Balzerani, Cronaca di un'attesa, ouvr. cité, p. 61.

110. B. Balzerani, Perché io, perché non tu, ouvr. cité, p. 80 (texte en italique original).

111. B. Balzerani, Lascia che il mare entri, ouvr. cité, p. 101.

\section{RÉSUMÉS}

L'étude vise à montrer que l'œuvre littéraire de Barbara Balzerani, motivée par le silence de la conflictualité politique et par les lacérations d'une histoire personnelle et collective, apparaît comme une tentative de recomposition d'une identité et d'une mémoire morcelées. Sans chercher à en masquer les sutures, le travail d'écriture de Balzerani s'apparente plutôt au tissage avec ses va-et-vient et ses échos qui réunissent temporalités et existences différentes. Dans cette perspective, les possibilités de résonnance des voix des subalternes, comme de celle de l'auteure, acquièrent une portée politique mais aussi vitale et existentielle.

Lo studio intende mostrare che l'opera letteraria di Barbara Balzerani, motivata dal silenzio della conflittualità politica e dalle lacerazioni di una storia personale e collettiva, appare come un tentativo di ricomposizione di un'identità e di una memoria frantumate. Senza cercare di mascherarne le suture, il lavoro di scrittura della Balzerani è piuttosto simile alla tessitura con il suo andirivieni e i suoi echi che riuniscono temporalità ed esistenze diverse. In questa prospettiva, le possibilità di risonanza delle voci dei subalterni, come di quella dell'autrice, acquisiscono una rilevanza politica ma anche vitale ed esistenziale.

The study aims to show that the literary work of Barbara Balzerani, motivated by the silence of political conflict and by the lacerations of a personal and collective history, appears as an 
attempt to recompose a fragmented identity and memory. Without seeking to hide its sutures, Balzerani's writing is more like weaving, with its comings and goings and its echoes that bring together different temporalities and existences. In this perspective, the possibilities of resonance of the voices of subordinates, as well as that of the author, acquire a political but also vital and existential significance.

INDEX

Mots-clés : Barbara Balzerani, autobiographies, recomposition, conflit politique

Parole chiave : Barbara Balzerani, autobiografie, ricomposizione, conflitto politico

Keywords : Barbara Balzerani, autobiographies, recomposition, political conflict

\section{AUTEUR}

\section{MARIE THIRION}

Université Grenoble Alpes

marie.thirion@univ-grenoble-alpes.fr 